\title{
Calidad del bosque de ribera del río EI Tunal, Durango, México; mediante la aplicación del índice QBR
}

\section{Quality of the riparian forest of El Tunal River, Durango, Mexico; trough the application of the QBR index}

\author{
Efraín Rodríguez-Téllez ${ }^{1}$ Pedro A. Domínguez-Calleros ${ }^{1}$ Marín Pompa-García ${ }^{1}$ José A. Quiroz- \\ Arratia ${ }^{1}$ \& María Elena Pérez López ${ }^{2}$ \\ ${ }^{1}$ Facultad de Ciencias Forestales, Universidad Juárez del Estado de Durango. Río Papaloapan Esq. Blvd. Durango s/n. Col. \\ Valle del sur. Durango, Durango. C.P. 34120 México. \\ ${ }^{2}$ Centro Interdisciplinario de Investigación para el Desarrollo Integral Regional. Instituto Politécnico Nacional. Sigma s/n, Fracc. \\ 20 de noviembre II, Durango, Durango. C.P. 34220. México. \\ efrainrodriguezt@hotmail.com
}

\begin{abstract}
RESUMEN
La evaluación de la condición ecológica de las riberas es un elemento importante en el estudio de los ríos alrededor del mundo. El objetivo del presente trabajo fue evaluar la calidad del bosque de ribera, desde un punto de vista estructural y funcional, en un tramo de 21 kilómetros del río El Tunal en Durango, México, mediante la aplicación del índice QBR. En el tramo estudiado se evaluaron seis sitios seleccionados por su representatividad, accesibilidad y heterogeneidad de su vegetación. En general, los resultados muestran un río con indicios importantes de deterioro; se encontraron dos sitios con calidad intermedia, dos sitios presentaron calidad mala y los dos restantes presentaron calidad pésima.
\end{abstract}

Palabras clave: QBR, El Tunal, México, bosque de ribera.

\begin{abstract}
The assessment of the ecological condition of the river banks is an important element in the rivers study around the world. The objective of this work was to evaluate the ecological condition of the riparian forest in a transect of $21 \mathrm{~km}$ of El Tunal river in Mexico with the QBR index. In the transect of study six sites were evaluated which were selected by the representation, possibilities of access and the heterogeneity of the vegetation. In general, the results have shown a river with important signs of deterioration; two sites results in an intermediate quality, two sites results in bad quality and two results in poor quality.
\end{abstract}

KeYwords: QBR, El Tunal, México, riparian forest.

\section{INTRODUCCIÓN}

El estudio de la condición ecológica de las riberas constituye un elemento relevante en el análisis integral de los ríos y arroyos. Éstos corresponden a uno de los tipos de ecosistemas más afectados por las actividades humanas, los cuales han sido muy alterados respecto al estado natural que debieran mantener (Kutschker et al. 2009). Algunas de las causas principales son: la regulación del caudal circulante, la introducción de especies exóticas, el cambio del uso de suelo y vegetación en las zonas aledañas; éstas han propiciado la disminución de la heterogeneidad ambiental natural en los paisajes ribereños e impactado negativamente en la biodiversidad y en los procesos ecológicos de los ríos (Ward 1989; Strayer et al. 2003, citados por Kutschker et al. 2009).

Algunas de las características de los ecosistemas riparios estriban en que son heterogéneos y complejos (Chovanec et al. 2000). Los ecosistemas ribereños como zonas de transición o interface efectúan la función de filtro al evitar la erosión de las riberas, amortiguar el ingreso de contaminantes y regular la temperatura y la entrada de luz. Lo anterior repercute en la estructura y la dinámica de los diferentes niveles tróficos (Kutschker et al. 2009). Existen múltiples interacciones entre los ecosistemas dulceacuícolas y los ecosistemas terrestres adyacentes, es evidente también 
que las alteraciones efectuadas en otras partes de la cuencavertiente pueden producir alteraciones importantes en el equilibrio natural de los ecosistemas riparios (Prat \& Ward 1994).

El desarrollo de métodos que permitan evaluar de manera rápida y eficaz la condición de los ecosistemas riparios ha despertado un alto interés en los últimos tiempos (Palma et al. 2009). Existen varios métodos para evaluar la condición biológica o de hábitat de los ríos y para evaluar la salud de los ríos y la integridad ecológica; pocos se han desarrollado específicamente para caracterizar hábitats ribereños. Sin embargo, se han efectuado esfuerzos para medir el grado de conservación de los ambientes riparios: e.g. se ha utilizado el River Habitat Survey (RHS), System for Evaluating Rivers for Conservation (SERCON), el índice RCE (Riparian, Channel and Environmental Inventory), el índice australiano ISC (Index of Stream Condition), el índice para corrientes de bajo gradiente sin marea, los índices de calidad de hábitats riparios usados en Protocolos de Evaluación Rápida o índices basados en aves acuáticas para evaluar condiciones de llanuras de inundación (Munné et al. 2003). Con la información generada por la aplicación de índices como el índice de calidad del bosque de ribera (QBR) se provee de elementos que permitan a los gestores ambientales construir planes o esquemas de manejo integral de los ecosistemas riparios, los cuales en algunos países son inexistentes.

El índice QBR ha sido aplicado con buenos resultados en ríos españoles (Ibero et al. 1996, Bonada et al. 2002), chilenos (Fernández et al. 2009, Palma et al. 2009), en ríos de Ecuador y Perú (Acosta et al. 2009) y en Argentina se ha utilizado en cursos de agua de la provincia de Tucumán (Kutschker et al. 2009). Analizando la necesidad de herramientas que permitan establecer en una forma rápida y eficaz la calidad de los bosques riparios, es necesario implementar en México metodologías que permitan establecer el estado ecológico que guardan los ecosistemas fluviales. En este estudio se plantea por primera vez la utilización del citado índice en México, el cual se seleccionó por los excelentes resultados que arroja, la sencillez en su aplicación y el bajo coste económico comparado con otras metodologías.

\section{MATERIALES Y MÉTODOS}

Área DE ESTUdio

El río El Tunal es el cauce principal de la cuenca San PedroMezquital, cuya superficie total es de $28.562 \mathrm{~km}^{2}$ y en ella se distribuyen principalmente cuatro comunidades vegetales: bosques de coníferas, praderas de chaparrales, selva seca y humedales costeros; este río drena sus aguas de noroeste a sur en el Estado de Durango, recibe aportaciones provenientes del estado de Zacatecas y desemboca en la parte norte del
Estado de Nayarit en una de las zonas más importantes de manglar de la República Mexicana, con una descarga total de 2,839 millones de metros cúbicos. El tramo estudiado de $21 \mathrm{~km}$ se localiza al suroeste de la ciudad de Durango en las coordenadas extremas, $23^{\circ} 57^{\prime} 38^{\prime \prime} \mathrm{N} ; 1^{\circ} 104^{\circ} 45^{\prime} 10^{\prime \prime} \mathrm{W}$ y $23^{\circ} 58^{\prime} 36^{\prime \prime} \mathrm{N}$; $104^{\circ} 35^{\prime} 56^{\prime \prime} \mathrm{W}$, y una altitud media de 1.889 $\mathrm{m}$ s.n.m. El río El Tunal tiene una gran importancia para los habitantes de la región ya que es una fuente fundamental para el abastecimiento de recursos hídricos para múltiples usos tales como el agrícola, pecuario, doméstico, urbano e industrial (Fig. 1). La zona de estudio se localiza en las estribaciones de la Provincia fisiográfica de la Sierra Madre Occidental; el clima es semiseco con lluvias en verano, presenta una temperatura media anual que varía entre los $12^{\circ}$ y $18^{\circ} \mathrm{C}$ y una precipitación de $561,4 \mathrm{~mm}$ anuales. Los tipos de vegetación aledaños a la zona son matorrales crasicaules, pastizales halófilos y matorrales xerófilos; la vegetación riparia se caracteriza por presentar un dosel alto de comunidades de Salix bonplandiana, Populus fremontii, Taxodium mucronatum, Fraxinus sp., Alnus acuminata, Cephalantus salicifolius, Juglans sp. y Ficus sp.

\section{MÉTODO DE TRABAJO Y VARIABLES A EVALUAR}

Para la elección del número de estaciones de muestreo se consideró la longitud del río en estudio y el detalle que se deseaba conseguir, siempre y cuando la distancia entre los sitios de muestreo no rebasase los $10 \mathrm{~km}$ (Acosta et al. 2009). A distancias mayores los aspectos que se describen no tendrían una continuidad y los valores resultantes serían muy variados y quedaría sin evaluar gran parte de la vegetación riparia existente. De esta forma se seleccionaron seis sitios a una equidistancia aproximada de 2,5 kilómetros.

Para evaluar la calidad de los bosques de ribera se utilizó el índice QBR (Munné et al. 1998, 2003). Este índice se centra en aspectos fundamentales de la vegetación ribereña, los cuales se agrupan en cuatro apartados: grado de cubierta de la zona de ribera, entendida como cubierta vegetal permanente, arbórea y arbustiva; estructura de la vegetación, es decir, estratos vegetales presentes y distribución de la vegetación; calidad de la cubierta, valorada a partir de la riqueza de especies arbóreas y arbustivas autóctonas (y dependiente, por tanto, del tipo geomorfológico de la zona de ribera) y grado de naturalidad del canal fluvial desde el punto de vista morfológico. Cada sección es valorada con una puntuación de 0 a 25 , no pudiendo sobrepasar estos límites y la sumatoria de la puntuación de las cuatro secciones varía entre 0 y 100 . La calidad resultante se distribuye en cinco rangos: $\geq 95$ : bosque de ribera sin alteraciones, calidad muy buena, estado natural; 75-90: bosque ligeramente perturbado, calidad buena; 55-70: inicio de alteración importante, calidad intermedia; 30-50: alteración fuerte, mala calidad; $\leq 25$ : degradación extrema, calidad pésima. Los formatos para recopilar la información para la aplicación del índice QBR pueden ser obtenidos de Jáimez-Cuéllar et al. (2002). 


\section{RESULTADOS}

Los valores del QBR en el tramo evaluado se muestran en la Tabla I. En el apartado de grado de cubierta de la zona de ribera se reportan valores bajos, los cuales oscilaron entre 0 y 5 . El porcentaje de cubierta vegetal de la zona de ribera osciló de menos de $10 \%$ hasta $50-80 \%$. La conectividad entre el bosque de ribera y la vegetación natural adyacente se presenta desde un 50\% hasta menos de $25 \%$.

En el apartado correspondiente a la estructura de la vegetación los valores oscilaron entre 0 y 15 . Se observó un recubrimiento de árboles que oscila desde $75 \%$ hasta menos de $10 \%$; los sitios 5 y 6 presentaron los valores más bajos en cuanto a recubrimiento arbóreo ya que en estos dos sitios la vegetación ha sido casi completamente eliminada por las actividades de extracción de materiales pétreos. La concentración de helófitos oscilo de buena a $25-75 \%$, excepto en los sitios 5 y 6 .

Respecto a la calidad de la cubierta, primero se determinó el tipo geomorfológico de cada uno se los sitios, lo cual definió su potencialidad para soportar una zona con vegetación; los sitios 1, 2 y 4 resultaron ser del tipo 2; el sitio 3 presentó una ribera de tipo 3 ; los sitios 5 y 6 presentaron una ribera de tipo 1 . Los valores en cuanto a calidad de la cubierta oscilaron entre 0 y 25 . En ninguno de los sitios se observó una continuidad total en sentido longitudinal en el bosque a lo largo del río. En todos los sitios se encontró más de 2 especies diferentes de arbustos, excepto en el sitio 5 en el que no se encontró un sotobosque establecido. En los sitios 1, 5 y 6 se apreciaron estructuras construidas por el hombre. La presencia de especies vegetales introducidas se observó en los sitios 1, 2 y 3; los vertidos de basura se registraron en los sitios 1,3 y 5 .

En el apartado correspondiente al grado de naturalidad del canal fluvial arrojó valores entre 0 y 25 . En los sitios 1 , 2,3 y 4 no se apreciaron modificaciones al canal natural del río, en el sitio 1 se apreció una gran estructura transversal al lecho del río (Presa Gral. Guadalupe Victoria), así como vestigios de una antigua presa derivadora; en el sitio 2 se encontraron vestigios de un antiguo puente; en el sitio 4 se encuentra una presa derivadora en operación. En los sitios 5 y 6 se observaron modificaciones al canal del río por la extracción de áridos.

Los valores totales del índice QBR para cada sitio oscilaron entre 0 y 60 , siendo notoria la ausencia de sitios con buena calidad o calidad muy buena, es decir sin alteraciones o en estado natural. Los sitios 2 y 3 presentan condiciones de inicio de alteración importante y se clasificaron como de calidad intermedia (Tabla I). Los sitios 1 y 4 presentaron alteración fuerte, clasificándose como de mala calidad. Los sitios 5 y 6 presentan una degradación extrema y se clasificaron como de calidad pésima.

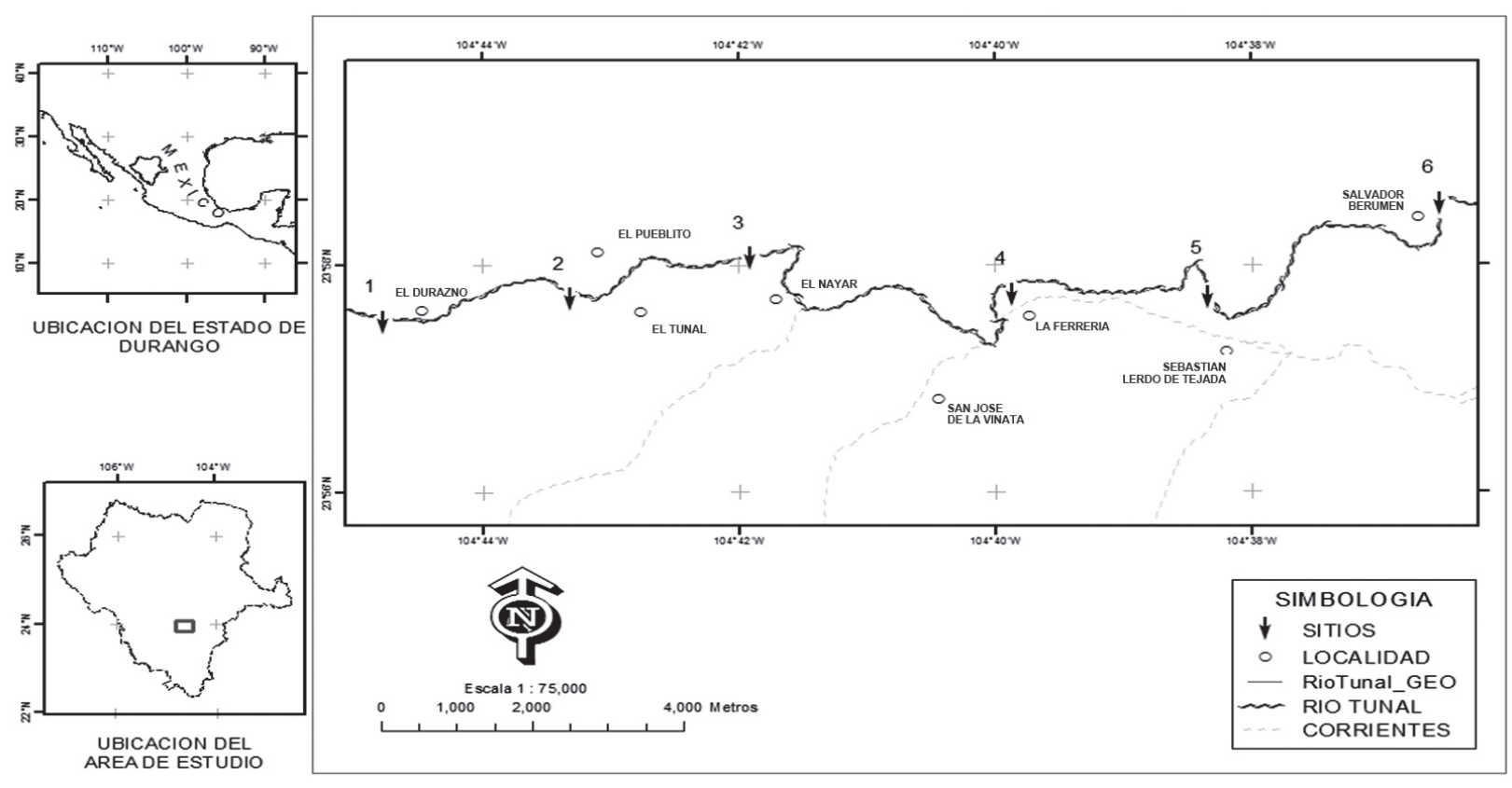

Figura 1. Localización del área de estudio.

Figure 1. Location of study area. 
Gayana Bot. 69(1), 2012

TABLA I. Valores para cada sección del QBR y QBR total para los seis sitios de evaluación.

TABLE I. Partial QBR Values for each four sections and total QBR for the six evaluation sites.

\begin{tabular}{cccccc}
\hline Sitio & $\begin{array}{c}\text { Grado de cubierta } \\
\text { De la zona de ribera }\end{array}$ & $\begin{array}{c}\text { Estructura de la } \\
\text { vegetacín en la zona } \\
\text { DE Ribera }\end{array}$ & $\begin{array}{c}\text { Calidad de la } \\
\text { Cubierta }\end{array}$ & $\begin{array}{c}\text { Grado de naturalidad } \\
\text { Del canal fluvial }\end{array}$ & QBR Total \\
\hline 1 & 0 & 15 & 15 & 15 & 45 \\
2 & 5 & 15 & 25 & 15 & 60 \\
3 & 0 & 10 & 20 & 25 & 45 \\
4 & 0 & 5 & 25 & 15 & 0 \\
5 & 0 & 0 & 0 & 0 & 15 \\
\hline
\end{tabular}

\section{DISCUSIÓN}

La aplicación del índice QBR para el establecimiento del grado de conservación de la vegetación ribereña del río El Tunal mostró una clara idea de las condiciones actuales que guarda este ecosistema. El índice resultó ser una herramienta económica y de rápida aplicación; además la información obtenida es de alta confiabilidad ya que relaciona aspectos fundamentales de la estructura y organización de los bosques riparios. Dado el origen de este índice, para ríos mediterráneos, es necesario tomar algunas consideraciones al momento de su aplicación en otros ecosistemas; por ejemplo si se quiere aplicar en otros ecosistemas que se distribuyen en la misma cuenca a la que pertenece este río, al momento de puntuar el apartado correspondiente a grado de cubierta de la zona de ribera, debe tenerse en cuenta que la vegetación es de tipo matorral semidesértico, la cual tiende a distribuirse de manera dispersa con una cobertura poco densa y el valor en este apartado del índice no debe subvalorarse (Suárez \& Vidal-Abarca 2000).

Otro aspecto a tenerse en cuenta en la aplicación del índice en otras cuencas es el número de especies diferentes de árboles autóctonos. En todos los sitios evaluados se encontraron más de 6 especies diferentes, por lo que éste sería un criterio que se sugiere modificar al tratarse de ríos de alta montaña, ya que aplicando este índice en otros tramos se podría sobrevalorar el índice en este apartado. En trabajos previos, Carrascosa \& Munné (2000) sugieren modificaciones al no penalizar la ausencia de un estrato arbóreo multiespecífico en ríos españoles de alta montaña.

En el tramo evaluado se apreciaron distintos efectos por algunas actividades humanas tales como asentamientos humanos, deforestación, pastoreo y agricultura que provocan una fuerte degradación de los ecosistemas riparios (Palma et al. 2009) que determinan las bajas puntuaciones del índice en el tramo evaluado. Esta misma situación ha sido reportada por Kutschker et al. (2009) que encontró que en los sitios sometidos a pastoreo se presentan situaciones de calidad de ribera desde intermedia a mala. Por otro lado, Merrit \& Cummins (1996) reportaron que el efecto de la ganadería se relaciona con la pérdida de la vegetación ribereña, que significa pérdida de protección, sombreo y aporte de materia orgánica gruesa.

Fue evidente en todos los sitios la existencia de áreas agrícolas que disminuyen la conectividad entre el bosque de ribera y ecosistema natural adyacente. Existen otras alteraciones tales como las apreciadas en los sitios 5 y 6 donde se realizan actividades de extracción de materiales pétreos, que ocasionan fuertes alteraciones geomorfológicas. Naiman \& Decamps (1993) señalan que éstas modifican el cauce, aumentan la sedimentación y la formación de bancos de arena reduciendo con esto la biodiversidad y alterando los hábitats ribereños. Las actividades humanas influyen directamente en la presencia de especies exóticas en el área; en casi todos los sitios evaluados se registró la presencia de especies alóctonas, las cuales afectan la naturalidad del río por el reemplazo paulatino de las especies nativas por parte de las especies introducidas.

Smith \& Armesto (2002) reportan que las estructuras transversales como las encontradas en los sitios 4, 5 y 6 además de contribuir a erosionar los suelos modifican la naturalidad del canal fluvial y actúan como rutas de invasión de especies exóticas; también funcionan como barreras que alteran los patrones de dispersión o migración de animales pudiendo mermar el tamaño de las poblaciones.

Dados los resultados obtenidos en el presente estudio, resulta fundamental el desarrollo de políticas y planes de manejo en el corto y mediano plazo de las riberas para 
asegurar la integridad ecológica del sistema fluvial del río El Tunal (Naiman et al. 2002, Palma et al. 2009) ya que, como se muestra en los resultados, ningún sitio presentó características de bosque de ribera con calidad buena o muy buena, por el contrario, la clasificación osciló entre pésima, mala e intermedia; por lo que un primer paso en la gestión del río El Tunal es la eliminación de los impactos que pudieran propicia la degradación de las riberas.

\section{AGRADECIMIENTOS}

Al M.C. Arnulfo Meléndez Soto por la elaboración de los mapas, a Everardo Valenzuela Hermosillo y Moises Morales Montes por su colaboración en la toma de datos en campo y a Luis Enrique Villa C., Rogelio Quiñones Scott, Maico Zapata Molina y Jaciel Rubio Cardoza por su entusiasta participación durante la elaboración de este estudio.

\section{BIBLIOGRAFÍA}

Acosta, R., B. Ríos, M. Rieradevall \& N. Prat. 2009. Propuesta de un protocolo de evaluación de la calidad ecológica de ríos andinos (CERA) y su aplicación a dos cuencas en Ecuador y Perú. Limnetica 28(1): 35-64.

Bonada, N., N. Prat, A. Munné, M. Plans, C. Solá, M. Álvarez, I. Pardo, G. Moyá, G. Ramon, M. Toro, S. Robles, J. Avilés, M.L. Suárez, M.R. Vidal-Abarca, A. Mellado, J.L. Moreno, C. Guerrero, S. Vivaz, M. Ortega, J. Casas, A. SÁnchez-Ortega, P. Jáimez-Cuellar \& J. Albatercedor. 2002. Intercalibración de la metodología GUADALMED. Selección de un protocolo de muestreo para la determinación del estado ecológico de los ríos mediterráneos. Limnetica 21: 13-33.

Carrascosa, V. \& A. Munne. 2000. Qualificació dels boscos de ribera andorrans. Adaptació de l'index QBR als d'alta muntanya. Habitats-Cenbtre de Biodiversitat (IEA) 1: 413.

Chovanec, A., P. Jäger, M. Jungwirth, V. Koller-Kreimel \& O. Moog. 2000. The Australian way of assessing the ecological integrity of running waters: a contribution to the EUWater Framework Directive. Hydrobiologia 422/423:445-452.

Fernández, L., J. Rau \& A. Arriagada. 2009. Calidad de la vegetación ribereña del río Maullín utilizando el índice
QBR. Gayana Botánica 66(2): 269-278.

Ibero, C., C. Alvarez, J.C. Blanco, J. Criada, A. Sánchez \& C. ViadA. 1996. Ríos de vida. El estado de conservación de las riberas fluviales en España. Sociedad Española de Ornitología, SEO-Birdlife. 45 pp.

Jáimez-Cuéllar, P., S. Vivas, N. Bonada, S. Robles, A. Mellado, M. Álvarez, J. Avilés, J. Casas, M. Ortega, I. Pardo, N. Prat, M. Rieradevall, C.E. Sáinz-Cantero, A. SánchezOrtega, M.L. Suárez, M. Toro, M.R. Vidal-Abarca, C. Zamora-Muñoz \& J. Alba-Tercedor. 2002. Protocolo GUADALMED (PRECE). Limnetica 21: 187-204.

KutschKer, A., C. Brand \& M.L. Miserendino. 2009. Evaluación de la calidad de los bosques de ribera en ríos del NO del Chubut sometidos a distintos usos de la tierra. Asociación Argentina de Ecología. Ecología Austral 19:19-34.

Merrit, R.W. \& K.W. Cummins. 1996. An introduction to the aquatic insects of North America. Kendall-Hunt. Dubuque. $862 \mathrm{pp}$.

Munné, A., C. SolÁ \& N. Prat. 1998. QBR: Un índice rápido para la evaluación de la calidad de los ecosistemas de ribera. Tecnología del Agua 175: 20-37.

Munné, A., N. Prat, C. Solá, N. Bonada \& M. Rieradevall. 2003. A simple field method for assessing the ecological quality of riparian habitat in rivers and streams: QBR index. Aquatic Conservation: Marine and Freshwater Ecosystems 13: $147-163$.

Naiman, R.J. \& H. Decamps. 1993. The role of riparian corridors in maintaining biodiversity. Ecological applications 2: 209-212.

Naiman, R.J., R.R. Bilby, D.E. Schindler \& J.M. Helfield. 2002. Pacific Salmon, nutrients, and the dynamics of Freshwater and Riparian Ecosystems. Ecosystems 5: 399-417.

Palma, A., R. Figueroa \& V.H. Ruiz. 2009. Evaluación de ribera y hábitat fluvial a través de los índices QBR e IHF. Gayana 73(1): 57-63.

Prat, N. \& J.N. Ward. 1994. The timed river. In: R. Margalef (ed.), Limnology now: a paradigm of planetary problems. Pp. 219-236. Elsevier Science B.V. Amsterdam.

Smith, C. \& J. Armesto. 2002. Importancia biológica de los bosques costeros de la Décima Región: el impacto de la Carretera Costera Sur. Ambiente y Desarrollo 23: 6-14.

SuÁrez, M.L. \& M.R. Vidal-Abarca. 2000. Aplicación del índice de calidad del bosque de ribera QBR, a los cauces fluviales de la cuenca del río Segura. Tecnología del agua 201: 33 45.

WARD, J.V. 1989. The four dimensional nature of lotic ecosystems. Journal of the North American Benthological Society 8(1): 2-8.

Recibido: 11.03 .11

Aceptado: 26.08.11 